\title{
GENERIC ROBUSTNESS OF SPECTRAL DECOMPOSITIONS
}

\author{
BY FLAVIO ABDENUR
}

\begin{abstract}
We prove that given a compact $n$-dimensional boundaryless manifold $M, n \geqslant 2$, there exists a residual subset $R$ of $\operatorname{Diff}^{1}(M)$ such that if $f \in R$ admits a spectral decomposition (i.e., the nonwandering set $\Omega(f)$ admits a partition into a finite number of transitive compact sets), then this spectral decomposition is robust in a generic sense (tame behavior). This implies a $C^{1}$-generic trichotomy that generalizes some aspects of a two-dimensional theorem of Mañé [Topology 17 (1978) 386-396].

Lastly, Palis [Astérisque 261 (2000) 335-347] has conjectured that densely in $\operatorname{Diff}^{k}(M)$ diffeomorphisms either are hyperbolic or exhibit homoclinic bifurcations. We use the aforementioned results to prove this conjecture in a large open region of $\operatorname{Diff}^{1}(M)$.
\end{abstract}

๑) 2003 Éditions scientifiques et médicales Elsevier SAS

RÉSUMÉ. - Nous montrons qu'étant donnée une variété compacte $M$ de dimension $n, n \geqslant 2$, il existe un sous-ensemble résiduel $R$ de $\operatorname{Diff}^{1}(M)$ tel que si $f \in R$ admet une décomposition spectrale (c'est-à-dire, $\Omega(f)$ admet une partition en un nombre fini d'ensembles compacts transitifs), alors cette décomposition spectrale est robuste dans un sens générique. Cela implique une trichotomie générique qui généralise certains aspects d'un théorème bi-dimensionnel de Mañé.

Enfin, Palis a conjecturé que dans un sous-ensemble dense de Diff ${ }^{1}(M)$, les difféomorphismes ou bien sont hyperboliques, ou bien admettent des bifurcations homoclines. Nous utilisons les résultats précédents pour prouver cette conjecture dans une grande région ouverte de $\operatorname{Diff}^{1}(M)$.

๑) 2003 Éditions scientifiques et médicales Elsevier SAS

\section{Statement of the results}

Throughout this paper, $M$ denotes a compact boundaryless manifold of dimension $n \geqslant 2$ and $\operatorname{Diff}^{1}(M)$ is the space of $C^{1}$-diffeomorphisms on $M$ with the usual topology. Given $f \in \operatorname{Diff}^{1}(M)$, the non-wandering set of $f$ is given by $\Omega(f) \equiv\{p \in M$ : given any open neighborhood $U$ of $p$ there is some $k \in \mathbb{N}$ such that $\left.f^{k}(U) \cap U \neq \emptyset\right\}$.

Given an open subset $U$ of $\operatorname{Diff}^{1}(M)$, a subset $R$ of $U$ is residual in $U$ if $R$ contains the intersection of a countable family of open and dense subsets of $U$; in this case $R$ is dense in $U$. A set $R \subset \operatorname{Diff}^{1}(M)$ is said to be residual if it is residual in all of $\operatorname{Diff}^{1}(M)$. A property (P) is residual or generic in $U$ if $(\mathrm{P})$ holds for all diffeomorphisms which belong to some residual subset of $U$; property (P) is said to be residual or generic if it is residual in all of $\operatorname{Diff}^{1}(M)$.

We begin by defining an object that plays a central role in the theory of hyperbolic systems:

Definition 1. - Let $f \in \operatorname{Diff}^{1}(M), p$ a periodic hyperbolic saddle of $f$. The homoclinic class of $f$ relative to $p$ is given by

$$
H(p, f)=c l\left[W^{s}(p) \pitchfork W^{u}(p)\right],
$$

ANNALES SCIENTIFIQUES DE L'ÉCOLE NORMALE SUPÉRIEURE

0012-9593/03/02/@ 2003 Éditions scientifiques et médicales Elsevier SAS. All rights reserved 
where $\pitchfork$ denotes points of transverse intersection of the invariant manifolds.

$H(p, f)$ is a transitive compact $f$-invariant subset of $\Omega(f) . H(p, f)$ is not necessarily hyperbolic, but when $f$ is Axiom A, its basic sets (see Definition 2 below) are hyperbolic homoclinic classes. In the absence of ambiguity, we may write $H(p)$ for $H(p, f)$.

Note that the definition above deliberately excludes periodic sinks/sources. This is convenient in the present context, but often it is useful to regard the orbits of sinks/sources as homoclinic classes.

Remark. - Henceforward we often write "sinks/sources" meaning "periodic sinks/sources".

Homoclinic classes are the natural candidates to replace hyperbolic basic sets in nonhyperbolic theory. Several recent papers (including Diaz, Pujals and Ures [10], Bonatti, Diaz and Pujals [5], Carballo and Morales [8], and Carballo, Morales and Pacifico [9]) explore their "hyperbolic-like" properties, many of which hold only for generic diffeomorphisms. This paper adopts a similar viewpoint.

It is well known that there exists a residual subset $R$ of $\operatorname{Diff}^{1}(M)$ such that if $f \in R$ has a finite number of sinks/sources, then the number of sinks/sources is constant in a $C^{1}$-neighborhood of $f$. (See for instance [16].) This means that no "new" sinks or sources arise in a neighborhood of $f$. Our first goal is to obtain an analogous result for homoclinic classes.

The aforementioned result on sinks/sources is an immediate consequence of an elementary topological fact: given a lower-semicontinuous correspondence from $\operatorname{Diff}^{1}(M)$ to the set of compact subsets of $M$, there exists a residual subset of $\operatorname{Diff}^{1}(M)$ where this correspondence is also upper-semicontinuous. Unfortunately, this fact cannot be applied in the same way to homoclinic classes, since they - unlike sinks/sources - are not necessarily isolated. However, other arguments from general topology yield the following result:

THEOREM A. - There exists a residual subset $R$ of $\operatorname{Diff}^{1}(M)$ such that if $f \in R$ only has finitely many distinct homoclinic classes $H\left(p_{1}, f\right), \ldots, H\left(p_{k}, f\right)$, then there exists a neighborhood $U$ of $f$ in $R$ such that if $g \in U$, then the only distinct homoclinic classes of $g$ are the continuations $H\left(p_{1}^{g}, g\right), \ldots, H\left(p_{k}^{g}, g\right)$ of the homoclinic classes of $f$.

Theorem A is our central result; all subsequent results in this paper essentially follow from it. Note that it does not require finitude of sinks/sources.

We now state some definitions regarding the structure of the non-wandering set:

DEFINITION 2. - A diffeomorphism $f \in \operatorname{Diff}^{1}(M)$ admits a spectral decomposition if $\Omega(f)=\Lambda_{1} \cup \cdots \cup \Lambda_{K_{f}}$, where the $\Lambda_{i}$ 's are pairwise disjoint compact transitive sets. (This means, roughly, that the dynamic of $f$ is "given by the orbits of a finite number of points.") The $\Lambda_{i}$ 's are called the basic sets of $f$.

Given a diffeomorphism $f \in \operatorname{Diff}^{1}(M)$ that admits a spectral decomposition, we say that $f$ has a dominated splitting if each basic set $\Lambda$ of $f$ either is hyperbolic or admits a dominated splitting.

In the hyperbolic context, the Spectral Decomposition Theorem tells us that an Axiom A diffeomorphism $\mathrm{f}$ always admits a spectral decomposition. If the diffeomorphism in question is also omega-stable (i.e., if it has no cycles), then this spectral decomposition is robust in a $C^{1}$-neighborhood of $f$. We want to extend the concept of robustness of spectral decompositions to the general, non-hyperbolic setting. We start with the statement of an easy proposition:

PROPOSITION A. - There exists a residual subset $S$ of $\operatorname{Diff}^{1}(M)$ such that if $f \in S$ then (a) and (b) are equivalent,

(a) $f$ admits a spectral decomposition, 
(b) $f$ has finitely many distinct homoclinic classes and finitely many sinks/sources.

Moreover, if $f \in S$ satisfies the conditions above, then $f$ has a dominated splitting and its spectral decomposition is of the form

$$
\Omega(f)=\Lambda\left(p_{1}\right) \cup \cdots \cup \Lambda\left(p_{K_{f}}\right),
$$

where $\Lambda(p)=H(p, f)$ if $p$ is a saddle and $\Lambda(p)$ is the $f$-orbit $\mathcal{O}(p)$ of $p$ if $p$ is a sink/source.

Definition 3.- Let $K_{1}$ and $K_{2}$ be compact subsets of $M$. The distance between $K_{1}$ and $K_{2}$ in the Hausdorff metric is given by

$$
d\left(K_{1}, K_{2}\right)=\inf \left\{\varepsilon>0: K_{1} \subset B_{\varepsilon}\left(K_{2}\right) \text { and } K_{2} \subset B_{\varepsilon}\left(K_{1}\right)\right\} .
$$

Definition 4. - Let $R \subset \operatorname{Diff}^{1}(M)$ be a residual set and $f \in R$ be a diffeomorphism that admits a spectral decomposition of the form $\Omega(f)=\Lambda\left(p_{1}\right) \cup \cdots \cup \Lambda\left(p_{K_{f}}\right)$ as in Proposition A. This spectral decomposition is $R$-robust (or, more generally, generically robust) if there exists an open neighborhood $U$ of $f$ in $R$ such that if $g \in U$, then $g$ admits a spectral decomposition $\Omega(g)=\Lambda\left(p_{1}^{g}\right) \cup \cdots \cup \Lambda\left(p_{K_{f}}^{g}\right)$ of the same form such that if $g_{i} \rightarrow f$ in the $C^{1}$ topology, $g_{i} \in R$, then $\Lambda\left(p_{j}^{g_{i}}\right) \rightarrow \Lambda\left(p_{j}\right)$ in the Hausdorff topology, for all $j \in\left\{1, \ldots, K_{f}\right\}$.

(This phenomenon has also been called tame behavior; in this terminology the case where there is a generic neighborhood where there is no spectral decomposition is called wild behavior; see for instance [4].)

Omega-stable diffeomorphisms admit generically robust spectral decompositions; in fact, their decompositions are robust in a much stronger, local, sense. We can think of generic robustness of spectral decompositions as a weak generalisation of omega stability. $C^{1}$-robustly transitive diffeomorphisms also trivially admit generically robust spectral decompositions. But how common is this phenomenon? Theorem B below provides a very complete answer to this question: generically speaking, spectral decompositions are always generically robust.

THEOREM B. - There exists a residual subset $R^{\prime}$ of $\operatorname{Diff}^{1}(M)$ such that if $f \in R^{\prime}$ admits a spectral decomposition, then this spectral decomposition is $R^{\prime}$-robust.

At this point it is relevant to mention a two-dimensional result of Mañé. In [16] he proved that, $C^{1}$-generically, surface diffeomorphisms either are omega-stable or have infinitely many sinks/sources. This result does not hold in higher dimensions, because of the existence of robustly nonhyperbolic and transitive diffeomorphisms in dimensions $\geqslant 3$ (see [15,2], and Bonatti and Viana [7] for examples). But we may still look for high-dimension analogues of Mañé's theorem, weakening the conclusion as needed.

It was in this spirit that [5] asked whether generic diffeomorphisms either admit spectral decompositions or have infinitely many sinks/sources. (The actual statement in [5], regarding generic diffeomorphisms that are "far from Newhouse's phenomenon", looks less general, but is easily seen to be equivalent to the statement in the previous sentence.) The example in [8], of a locally residual subset of $\operatorname{Diff}^{1}(M)$ where there are only 3 sinks/sources but infinitely many homoclinic classes (and no spectral decomposition), gives a negative answer to this question. But we can obtain a $C^{1}$-generic trichotomy in high dimensions by adding a third alternative:

COROLlaRY B.1. - There exists a residual subset $R^{+} \subset \operatorname{Diff}^{1}(M)$ such that if $f \in R^{+}$, $n \geqslant 3$, then $f$ satisfies one and only one of the following conditions:

(a) $f$ admits an $R^{+}$-robust spectral decomposition $\Omega(f)=\Lambda\left(p_{1}\right) \cup \cdots \cup \Lambda\left(p_{K_{f}}\right)$ with a dominated splitting, 
(b) $f$ has an infinite number of sinks/sources (and therefore admits no spectral decomposition),

(c) $f$ admits no spectral decomposition, has only a finite number of sinks/ sources, and has an infinite number of pairwise disjoint homoclinic classes.

Corollary B.1 is currently the best high-dimensional analogue of Mañé's theorem. In dimension 2, Mañé's theorem says that we generically have either a pathology (infinite number of sinks/sources) or a robust spectral decomposition with a hyperbolic splitting; in dimension $\geqslant 3$, Corollary B.1 says that we generically have either a pathology (infinite number of pairwise disjoint homoclinic classes or infinite number of sinks/sources) or a generically robust spectral decomposition with a dominated splitting. Furthermore, in [1] it is shown that this dominated splitting exhibits a weak form of hyperbolicity called volume partial hyperbolicity (see [5] for the definition).

We remark that examples in [3] and [8] respectively show that neither alternative (b) nor alternative (c) can be removed from the statement of Corollary B.1 for dimensions $n \geqslant 4$. Alternative (a) of course cannot be removed due to the existence of omega-stable diffeomorphisms. In this sense the trichotomy above is optimal: none of the three alternatives may be removed from the statement.

The next corollary is a simple but useful consequence of Theorem B and the generic "nocycles" result of [9]:

COROLlaRY B.2. - There exists a residual subset $R_{+} \subset \operatorname{Diff}^{1}(M)$ such that if $f \in R_{+}$ admits a spectral decomposition $\Omega(f)=\Lambda\left(p_{1}\right) \cup \cdots \cup \Lambda\left(p_{K_{f}}\right)$ as in Proposition A, then given pairwise disjoint open neighborhoods $W_{1}, \ldots, W_{K_{f}}$ of $\Lambda\left(p_{1}\right), \ldots, \Lambda\left(p_{K_{f}}\right)$ respectively, there exists an open neighborhood $U$ of $f$ in $\operatorname{Diff}^{1}(M)$ with the following properties:

(i) if $g \in U$, then $\Omega(g) \subset\left(W_{1} \cup \cdots \cup W_{K_{f}}\right)$,

(ii) if $g \in\left(U \cap R_{+}\right)$, then $\left(\Omega(g) \cap W_{j}\right)=\Lambda\left(p_{j}{ }^{g}\right)$ for all $j \in\left\{1, \ldots, K_{f}\right\}$.

Palis [17] has conjectured that there exists a dense subset $D$ of $\operatorname{Diff}^{k}(M)$ such that if $f \in D$, then $f$ either is hyperbolic or exhibits homoclinic tangencies or heterodimensional cycles. By heterodimensional cycle we mean a heteroclinic cycle between periodic saddles of different stability indices. Homoclinic tangencies and heterodimensional cycles are both examples of a broader class of phenomena called homoclinic bifurcations (see [20], pp. 133 and 134). Roughly speaking, Palis has conjectured that, globally, homoclinic bifurcations are the only obstructions to hyperbolicity.

The 2-dimensional $C^{1}$-version of this conjecture has been completely proven by Pujals and Sambarino [19], but results in higher dimensions have been restricted to special cases, like robustly transitive diffeomorphisms (in which case it essentially follows from results by Mañé [16] and from Hayashi's Connecting Lemma [13]; see also Bonatti, Diaz, Pujals and Rocha [6]). The last goal of this paper is to prove this conjecture in a large region of $\operatorname{Diff}^{1}(M)$, where $M$ has arbitrary dimension.

In this paper we work towards the following $C^{1}$-generic version of the conjecture (which is actually somewhat stronger than the form above):

CONJECTURE (Palis). - There exists a residual subset $R$ of $\operatorname{Diff}^{1}(M)$ such that if $f \in R$, then $f$ is either omega-stable or approached by diffeomorphisms exhibiting homoclinic tangencies or heterodimensional cycles.

We note that by Pugh's General Density Theorem [18] combined with item 6 of Theorem A of [9], generically a diffeomorphism is omega-stable if and only if its non-wandering set is hyperbolic. The next result proves the conjecture above in the case of diffeomorphisms with finitely many homoclinic classes. 
THEOREM C. - There exists a residual subset $R$ of $\operatorname{Diff}^{1}(M)$ such that if $f \in R$ has finitely many homoclinic classes and finitely many sinks/sources, then $f$ satisfies (1) or (2) but not both:

(1) $f$ is omega-stable (i.e., Axiom A with no cycles),

(2) $f$ is nonhyperbolic and approached by diffeomorphisms which exhibit heterodimensional cycles.

This result follows from Corollary B.2 combined with results from [12] and from [13].

We note that the analogues of Theorems $\mathrm{A}$ and $\mathrm{B}$ hold for $C^{1}$-vector fields on manifolds of dimensions three and higher, with the same proofs.

\section{Technical preliminaries I}

We now state some results that will be used in the next section.

THEOREM 0 [5]. - There exists a residual subset $R^{*} \subset \operatorname{Diff}^{1}(M)$ such that if $f \in R^{*}$ has finitely many sinks/sources, then every homoclinic class (which is not the orbit of a sink/source) of $f$ has a dominated splitting.

THEOREM 1 [18]. - There exists a residual subset $R_{1} \subset \operatorname{Diff}^{1}(M)$ such that if $f \in R_{1}$, then $\Omega(f)=\operatorname{cl}(\operatorname{Per}(f))$ and $\operatorname{Per}(f)=\operatorname{Per}_{h}(f)$, where $\operatorname{Per}_{h}(f)$ denotes the set of hyperbolic periodic points of $f$.

THEOREM 2 [9]. - There exists a residual subset $R_{2} \subset \operatorname{Diff}^{-1}(M)$ such that if $f \in R_{2}$, then given any two saddles $p, q \in \operatorname{Per}(f)$, we have either

$$
H(p, f)=H(q, f) \quad \text { or } \quad H(p, f) \cap H(q, f)=\emptyset .
$$

That is, for diffeomorphisms in $R_{2}$, distinct homoclinic classes are disjoint. Furthermore, if $f \in R_{2}$ then there are no cycles among homoclinic classes of $f$.

THEOREM 3 [3]. - There exists a residual subset $R_{3} \subset \operatorname{Diff}^{1}(M)$ such that if $f \in R_{3}$ and $p, q \in \operatorname{Per}(f)$ are saddles that belong to the same transitive set $\Lambda$, then $H(p, f)=H(q, f)$.

\section{Proof of Proposition A}

We prove a slightly more general statement than Proposition A:

Proposition A'. - There exists a residual subset $R_{0} \subset \operatorname{Diff}^{1}(M)$ such that if $f \in R_{0}$, then $f$ satisfies one and only one of the following conditions:

(a) $\Omega(f)=\Lambda\left(p_{1}\right) \cup \cdots \cup \Lambda\left(p_{K_{f}}\right)$, where $p_{i} \in \operatorname{Per}_{h}(f)$ and the $\Lambda$ 's are compact transitive pairwise disjoint sets given by $\Lambda(p)=H(p, f)$ if $p$ is a saddle and $\Lambda(p)=\theta(p)$ if $p$ is a sink/source.

(b) $f$ has an infinite number of sinks/sources (and therefore admits no spectral decomposition).

(c) $f$ admits no spectral decomposition, has only a finite number of sinks/ sources, and has an infinite number of pairwise disjoint homoclinic classes.

Proof. - Let $R^{*}, R_{1}, R_{2}$, and $R_{3}$ respectively be as in Theorem 0 , Theorem 1, Theorem 2, and Theorem 3 above. Consider the residual $R_{0}$ given by $R_{0}=R^{*} \cap R_{1} \cap R_{2} \cap R_{3}$. Assume $f \in R_{0}$ has only a finite number of distinct (and therefore disjoint, by Theorem 2 ) homoclinic classes and a finite number of periodic sinks/sources. That is, let $f$ satisfy neither (b) nor (c). Let $H\left(p_{1}\right), \ldots, H\left(p_{k}\right)$ be the distinct homoclinic classes of $f$, and let $\theta\left(q_{1}\right), \ldots, \theta\left(q_{s}\right)$ be the orbits of its periodic sinks/sources. 
Then by Theorem 1 we have

$$
\begin{aligned}
& H\left(p_{1}\right) \cup \cdots \cup H\left(p_{k}\right) \cup \theta\left(q_{1}\right) \cup \cdots \cup \theta\left(q_{s}\right) \\
& \quad=c l\left[H\left(p_{1}\right) \cup \cdots \cup H\left(p_{k}\right) \cup \theta\left(q_{1}\right) \cup \cdots \cup \theta\left(q_{s}\right)\right] \\
& \quad=c l(\operatorname{Per}(f))=c l\left(\operatorname{Per}_{h}(f)\right)=\Omega(f),
\end{aligned}
$$

where the unions are pairwise disjoint. Therefore $f$ satisfies (a).

What we must prove now is that if $f \in R_{0}$ does not satisfy (a), then $f$ does not admit a spectral decomposition. Let $f \in R_{0}$ satisfy (b). Then $f$ has an infinite number of periodic sinks/sources and therefore clearly does not admit any spectral decomposition. If $f$ has infinitely many pairwise disjoint homoclinic classes and only a finite number of sinks/sources, assume $f$ has infinitely many pairwise disjoint homoclinic classes but admits a spectral decomposition $\Omega(f)=\Lambda_{1} \cup \cdots \cup \Lambda_{k}$. Then each homoclinic class of $f$ is contained in one of the $\Lambda$ 's, which are by assumption transitive. But by Theorem 3 if two periodic saddles of $f$ are contained in the same transitive set, then their homoclinic classes coincide. Therefore $f$ has at most $k$ distinct homoclinic classes, a contradiction. We conclude that $f \in R_{0}$ admits a spectral decomposition if and only if $f$ satisfies (a).

\section{Technical preliminaries II}

Again, some technical preliminaries:

Definition 5. - Let $p$ be a periodic saddle of $f \in \operatorname{Diff}^{1}(M)$. Then $H(p)$ varies $C^{1}$-continuously at $f$ if the map $g \rightarrow H\left(g, p^{g}\right)$ is continuous with respect to the $C^{1}$ topology on $\operatorname{Diff}^{1}(M)$ and to the Hausdorff topology in the space of compact subsets of $M$.

The next theorem is a well-known property of homoclinic classes. It follows from the two following facts: (i) any given homoclinic class $H(p, f)$ varies lower semi-continuously in the Hausdorff topology when $f$ is perturbed; and (ii) generically any set-valued map that is lower semi-continuous in the Hausdorff topology is also upper semi-continuous.

THEOREM 4. - There exists a residual subset $R_{4} \subset \operatorname{Diff}^{1}(M)$ such that if $f \in R_{4}$ and $p$ is a saddle of $f$, then $H(p)$ varies $C^{1}$-continuously at $f$.

THEOREM 5 [16]. - There exists a residual subset $R_{5} \subset \operatorname{Diff}^{1}(M)$ such that if $f \in R_{5}$ has finitely many sinks/sources $q_{1}, \ldots, q_{k_{f}}$ then there exists a neighborhood $U$ of $f$ in $\operatorname{Diff}^{1}(M)$ such that if $g \in U$, then the only sinks/sources of $g$ are the continuations $q_{1}{ }^{g}, \ldots, q_{k_{f}}{ }^{g}$ of the sinks/sources of $f$.

Definition 6. - A subset $S$ of a topological space $X$ is meager if $S^{c}$ is residual in $X$. Note that if $R \subset X$ is residual and $S \subset X$ is non-meager, then $R \cap S \neq \emptyset$.

Topological Lemma. - Let $X$ be a Baire topological space and $\Gamma: X \rightarrow \mathbb{N}$ a lower semicontinuous map. Then there exists a residual subset $N$ of $X$ such that $\Gamma$ is locally constant at each point of $N$.

\section{Proof of Theorem $A$}

Theorem A follows from Propositions 1 and 2 below.

Proposition 1. - Let $U \subset \operatorname{Diff}^{1}(M)$ be open and $R$ be a residual subset of $U$ such that if $f \in R$, then $f$ only has finitely many distinct homoclinic classes $H\left(f, p_{1}\right), \ldots, H\left(f, p_{k_{f}}\right)$. Then 
there exists a residual subset $N$ of $U$ such that given $f \in N$, there exists an open neighborhood $W$ of $f$ in $U$ such that if $g \in(W \cap N)$ then the only distinct homoclinic classes of $g$ are the continuations $H\left(g, p_{1}{ }^{g}\right), \ldots, H\left(g, p_{k_{f}}{ }^{g}\right)$ of the homoclinic classes of $f$.

Proof. - Let $R$ be as in the statement, $R_{2}$ as in Theorem 2, and $R_{4}$ as in Theorem 4. Consider $S=R_{2} \cap R_{4} \cap R$. S is generic in $U$. If $f \in S$, then $f \in R$, and therefore $f$ only has finitely many homoclinic classes $H\left(f, p_{1}\right), \ldots, H\left(f, p_{k_{f}}\right)$, which are pairwise disjoint by Theorem 2 . By Theorem 4, each such homoclinic class varies continuously at each $f \in S$.

Consider now the map $\Gamma: S \rightarrow \mathbb{N}$ given by

$$
\Gamma(f) \equiv \#\{\text { distinct homoclinic classes of } f\} .
$$

Note that given any $f \in S$, then $f$ only has a finite number of distinct homoclinic classes $H\left(f, p_{1}\right), \ldots, H\left(f, p_{k_{f}}\right)$, which must be pairwise disjoint by Theorem 2 . Therefore for $g \in S$ sufficiently close to $f$ the periodic saddles $p_{1}, \ldots, p_{k_{f}}$ have continuations, and since their corresponding homoclinic classes $H\left(p_{1}\right), \ldots, H\left(p_{k_{f}}\right)$ are pairwise disjoint and vary continuously at $f$, it follows that for $g$ sufficiently close to $f$, the homoclinic classes $H\left(g, p_{1}{ }^{g}\right), \ldots, H\left(g, p_{k_{f}}{ }^{g}\right)$ are still pairwise disjoint. Thus $\Gamma$ is a lower semi-continuous map on $S$ : elements of $S$ which are sufficiently close to $f$ must have at least as many distinct homoclinic classes as $f$.

We can now apply the Topological Lemma to $\Gamma$, obtaining a residual subset $N$ of $S$ (and therefore of $U$ ) where $\Gamma$ is locally constant, which is the property we wanted.

We now state and prove a lemma that will be used to prove Proposition 2.

Lemma 1. - Consider an open set $U$ in $\operatorname{Diff}^{-1}(M)$ such that $D=\{f \in U: f$ has an infinite number of pairwise disjoint homoclinic classes, all of which vary continuously at $f\}$ is dense in $U$. Then $D^{*}=\{f \in U: f$ has an infinite number of pairwise disjoint homoclinic classes $\}$ is residual in $U$.

Proof. - Let $D_{j}=\{f \in U: f$ has at least $j$ disjoint homoclinic classes $\}$. Then $\operatorname{int}\left(D_{j}\right)$ contains $D$ (because of the continuous variation of the homoclinic classes at elements of $D$ ) and is therefore open and dense in $U$. Therefore $D^{*} \supset \bigcap_{j \in \mathbb{N}} \operatorname{int}\left(D_{j}\right)$ is residual in $U$.

PROPOSITION 2. - There exists a residual subset $R_{6} \subset \operatorname{Diff}^{1}(M)$ such that if $f \in R_{6}$ only has finitely many distinct homoclinic classes, then there exists a neighborhood $U$ of $f$ in $R_{6}$ such that if $g \in U$ then $g$ only has finitely many homoclinic classes.

Proof. - Let $S$ be the residual subset of $\operatorname{Diff}^{1}(M)$ given by $S=R_{4}$ as in Theorem 4 above. We can write $S=S_{f i n} \cup S_{\infty}$, where $S_{f i n}$ denotes the elements of $S$ which have finitely many homoclinic classes and $S_{\infty} \equiv S \backslash S_{f i n}$.

If $\operatorname{cl}\left(S_{\infty}\right) \cap S_{\text {fin }}$ is a meager subset of $S$, where $c l$ denotes closure relative to $S$, then Proposition 2 clearly holds, since in this case

$$
R_{6} \equiv S \backslash\left(\operatorname{cl}\left(S_{\infty}\right) \cap S_{\text {fin }}\right)
$$

is residual and has the desired property. Let us now prove the meagerness of $\operatorname{cl}\left(S_{\infty}\right) \cap S_{f i n}$.

We have

$$
\operatorname{cl}\left(S_{\infty}\right) \cap S_{\text {fin }}=\left[\operatorname{int}\left(\operatorname{cl}\left(S_{\infty}\right)\right) \cap S_{\text {fin }}\right] \cup\left[\left(\operatorname{cl}\left(S_{\infty}\right) \backslash \operatorname{int}\left(\operatorname{cl}\left(S_{\infty}\right)\right)\right) \cap S_{\text {fin }}\right]
$$


where int denotes interior relative to $S$. Now, $\left(\operatorname{cl}\left(S_{\infty}\right) \backslash \operatorname{int}\left(\operatorname{cl}\left(S_{\infty}\right)\right)\right) \cap S_{\text {fin }}$ is meager because $\operatorname{cl}\left(S_{\infty}\right) \backslash \operatorname{int}\left(\operatorname{cl}\left(S_{\infty}\right)\right)$ is a closed set with empty interior. Therefore we must only show that $\operatorname{int}\left(\operatorname{cl}\left(S_{\infty}\right)\right) \cap S_{f i n}$ is meager.

By definition $\operatorname{int}\left(\operatorname{cl}\left(S_{\infty}\right)\right)$ is an open subset of $S$ where $S_{\infty}$ is dense. Therefore there exists some $U$ open in $\operatorname{Diff}^{1}(M)$ such that $U \cap S=\operatorname{int}\left(\operatorname{cl}\left(S_{\infty}\right)\right) . S_{\infty}$ is dense in $U$, and by Theorem 4 homoclinic classes vary continuously at members of $S_{\infty}$. Therefore applying Lemma 1 to $U$, we have that $S_{\infty}$ is residual in $U$.

Assume that $\operatorname{int}\left(\operatorname{cl}\left(S_{\infty}\right)\right) \cap S_{\text {fin }}$ is non-meager. Then there must be nonempty intersection between $S_{\infty}$, which is residual in $U$, and $S_{f i n}$, which is non-meager in $U$; this is of course absurd. Hence $\operatorname{int}\left(\operatorname{cl}\left(S_{\infty}\right)\right) \cap S_{f i n}$ is meager, as we wished to show.

We can now prove Theorem A:

Proof of Theorem A. - Consider the residual subset $R_{6}$ of $\operatorname{Diff}^{1}(M)$ given by Proposition 2 and let $S_{6} \equiv\left\{g \in R_{6}: g\right.$ only has a finite number of homoclinic classes $\}$. Then for each $g \in S_{6}$ there is some open neighborhood $U_{g}$ of $g$ in $R_{6}$ such that every element of $U_{g}$ also has a finite number of homoclinic classes. Now by Proposition 1 each $U_{g}$ contains some smaller neighborhood $W_{g}$ of $g$ such that the homoclinic classes of each element of $W_{g}$ are precisely the continuations of the homoclinic classes of $g$. Now the set $R$ given by

$$
R \equiv\left(R_{6} \backslash S_{6}\right) \cup \bigcup_{g \in S_{6}} W_{g}
$$

is residual in $\operatorname{Diff}^{1}(M)$ and clearly satisfies the desired conditions.

\section{Proof of Theorem B and its corollaries}

We now obtain Theorem B as a consequence of Theorem A:

Proof of Theorem B.-Consider the residual subsets $R_{5}$ as in Theorem 5, $R_{0}$ as in Proposition $\mathrm{A}^{\prime}$, and $R$ as in Theorem A. Let $R^{\prime}$ be the residual set given by $R^{\prime}=R_{0} \cap R \cap R_{5}$.

Given $f \in R^{\prime}$ with a spectral decomposition, by Proposition $\mathrm{A}^{\prime}$ we have

$$
\Omega(f)=\Lambda\left(p_{1}\right) \cup \cdots \cup \Lambda\left(p_{K_{f}}\right)
$$

as in Proposition A. Let us rename this spectral decomposition by writing

$$
\Omega(f)=H\left(p_{1}\right) \cup \cdots \cup H\left(p_{L_{f}}\right) \cup \theta\left(q_{L_{f}+1}\right) \cup \cdots \cup \theta\left(q_{K_{f}}\right),
$$

where $p_{i}$ is a periodic saddle with $H\left(p_{i}\right)$ its homoclinic class if $i \in\left\{1, \ldots, L_{f}\right\}$ and $q_{i}$ is a sink/source with orbit $\theta\left(q_{i}\right)$ if $i \in\left\{L_{f}+1, \ldots, K_{f}\right\}$. We want to show that this spectral decomposition persists in a neighbourhood of $f$ in $R^{\prime}$.

By Theorem A there exists a neighborhood $U_{1}$ of $f$ in $R$ (and therefore in $R^{\prime}$ ) such that if $g \in U_{1}$ then the only homoclinic classes of $g$ are the continuations $H\left(p_{1}{ }^{g}\right), \ldots, H\left(p_{L_{f}}{ }^{g}\right)$ of the classes of $f$. By Theorem 5 there is a neighborhood $U_{2}$ of $f$ in $\operatorname{Diff}^{1}(M)$ such that if $g \in U_{2}$ then the only sinks/sources of $g$ are the continuations $\theta\left(q_{L_{f}+1}{ }^{g}\right), \ldots, \theta\left(q_{K_{f}}{ }^{g}\right)$ of the sinks/sources of $f$.

Let $U \equiv U_{1} \cap U_{2}$. If $g \in U$ it follows that the only homoclinic classes of $g$ are $H\left(p_{1}{ }^{g}\right), \ldots$, $H\left(p_{L_{f}}{ }^{g}\right)$ and the only sinks/sources are $\theta\left(q_{L_{f}+1}{ }^{g}\right), \ldots, \theta\left({q_{K_{f}}}^{g}\right)$. By Proposition $\mathrm{A}^{\prime}, g$ admits 
the spectral decomposition

$$
\Omega(g)=H\left(p_{1}{ }^{g}\right) \cup \cdots \cup H\left({p_{L_{f}}}^{g}\right) \cup \theta\left({q_{L_{f}+1}}^{g}\right) \cup \cdots \cup \theta\left({q_{K_{f}}}^{g}\right) .
$$

Moreover, the basic sets of this decomposition vary continuously at $f$ since $R^{\prime}$ is contained in $R$ of Theorem A, which in turn is contained in $R_{4}$ of Theorem 4 . Hence we have proven that the spectral decomposition of $f$ is $R^{\prime}$-robust.

Proof of Corollary B.1. - Let $R^{+}=R^{*} \cap R_{0} \cap R^{\prime}$, with $R^{*}$ as in Theorem $0, R_{0}$ as in Proposition $\mathrm{A}^{\prime}$, and $R^{\prime}$ as in Theorem $\mathrm{B}$. Then $R^{+}$immediately satisfies the desired conditions.

Proof of Corollary B.2. - Let $R_{+}=R^{\prime} \cap R_{2}$, with $R^{\prime}$ as in Theorem B and $R_{2}$ as in Theorem 2 . Assume that $f \in R_{+}$admits a spectral decomposition $\Omega(f)=\Lambda\left(p_{1}\right) \cup \cdots \cup \Lambda\left(p_{K_{f}}\right)$. By Theorem 2 there are no cycles among homoclinic classes of $f$. Since there can be no cycles involving the orbits of sinks/sources, it follows that there are no cycles among the basic sets of $f$. By a well-known result (see for instance Theorems 2.3 and 3.11 of [21]), this implies that there are no $\Omega$-explosions at $f$. Also, since $R_{+} \subset R^{\prime} \subset R_{4}$ of Theorem 4, all homoclinic classes of $f$ vary continuously at $f$.

Let $W_{1}, \ldots, W_{K_{f}}$ be pairwise disjoint open neighborhoods of $\Lambda\left(p_{1}\right), \ldots, \Lambda\left(p_{K_{f}}\right)$ respectively. Then $W=W_{1} \cup \cdots \cup W_{K_{f}}$ contains $\Lambda\left(p_{1}\right) \cup \cdots \cup \Lambda\left(p_{K_{f}}\right)=\Omega(f)$, so since there are no $\Omega$-explosions at $f$, it follows that there exists an open neighborhood $U$ of $f$ in $\operatorname{Diffl}^{1}(M)$ such that if $g \in U$ then $\Omega(g) \subset W$. Condition (i) is proven.

We now prove condition (ii). If $g \in\left(U \cap R_{+}\right)$then given any $i \in\left\{1, \ldots, K_{f}\right\}$, we have that $\left(W_{i} \cap \Lambda\left(p_{i}\right)\right)$ contains $\Lambda\left(p_{i}\right)$ but does not intersect any other $\Lambda\left(p_{j}\right)$, therefore

$$
\left(W_{i} \cap \Lambda\left(p_{i}\right)\right)=\Lambda\left(p_{i}\right) .
$$

\section{Technical preliminaries III}

We now list some properties and results that will be used in the next section.

DEFINITION 7.-Given a periodic hyperbolic point $p$, its stability index is the dimension of its stable manifold $W^{s}(p)$. We denote the stability index of $p$ by ind $(p)$.

FRANKS' LEMMA [11]. - Consider $f \in \operatorname{Diff}^{1}(M)$, an $f$-invariant finite set $F$, and an $\varepsilon$-perturbation $A$ of $\left.D f\right|_{F}$. Then there exists an $\varepsilon$-perturbation $g$ of $f$ such that $\left.g\right|_{F}=\left.f\right|_{F}$ and $\left.D g\right|_{F}=A$.

Note that in the special case where $F$ is the orbit of a nonhyperbolic periodic point of $f$, this means that for all $\varepsilon$ there exist two $\varepsilon$-perturbations $g^{\prime}$ and $g^{\prime \prime}$ of $f$ such that $F$ respectively becomes the orbit of hyperbolic periodic points $p^{\prime}$ and $p^{\prime \prime}$ with different indices.

THEOREM 6 [12]. - Let $\Im^{1}(M)=\left\{f \in \operatorname{Diff}^{1}(M)\right.$ : there exists an open neighborhood $U$ of $f$ in $\operatorname{Diff}^{1}(M)$ such that if $g \in U$, then all periodic points of $g$ are hyperbolic $\}$. Then all members of $\Im^{1}(M)$ are omega-stable.

CONNECTING LeMma [13]. - Consider $f \in \operatorname{Diff}^{1}(M)$ with hyperbolic periodic points $p$ and q. Assume that there are sequences of points $\left(x_{j}\right)$ and natural numbers $\left(k_{j}\right)$ such that

$$
\left(x_{j}\right) \rightarrow p^{s} \in W^{s}(p) \backslash\{p\} \quad \text { and } \quad f^{k_{j}}\left(x_{j}\right) \rightarrow q^{u} \in W^{u}(q) \backslash\{q\} .
$$

Then given any $\varepsilon>0$ there exists an $\varepsilon$-perturbation $g$ of $f$ such that $W^{s}\left(p^{g}\right) \cap W^{u}\left(q^{g}\right) \neq \emptyset$. 


\section{Proof of Theorem C}

We first prove a lemma, whose proof follows that of Lemma 3.3 of Diaz, Pujals and Ures [10], and then use it to prove Theorem $\mathrm{C}$.

LEMMA 2. - Let $\Lambda(p)$ be the homoclinic class of a periodic saddle $p$ of $f \in \operatorname{Diff}^{1}(M)$. Assume that there exist a neighborhood $U$ of $f$ in $\operatorname{Diff}^{1}(M)$, a residual subset $R$ of $U$ containing $f$, and a periodic saddle $q$ of $f$ with ind $(q) \neq i n d(p)$ such that if $g \in R$, then $\Lambda\left(p^{g}\right)$ contains the continuation $q^{g}$ of $q$. Then, given any $\varepsilon>0$, there exists an $\varepsilon$-perturbation $f^{\prime}$ of $f$ such that there is a heterodimensional cycle between $p^{f^{\prime}}$ and $q^{f^{\prime}}$.

Proof. - By hypothesis $q$ and $p$ have different indices. Assume $\operatorname{ind}(p)>\operatorname{ind}(q)$; the other case is identical if we switch the roles of $p$ and $q$. We first want to create an intersection between $W^{s}(p)$ and $W^{u}(q)$.

Let $f$ be as in the statement and $\varepsilon>0$. By transitivity, there exists some point $x$ in $\Lambda(p)$ whose orbit is dense in $\Lambda(p)$. This means that there are sequences of increasing natural numbers $\left(m_{j}\right)$ and $\left(d_{j}\right)$ with $d_{j}>m_{j}$ such that

$$
f^{m_{j}}(x) \rightarrow p \quad \text { and } \quad f^{d_{j}}(x) \rightarrow q
$$

By considering fundamental domains $D^{s}$ of $p$ in $W^{s}{ }_{l o c}(p)$ and $D^{u}$ of $q$ in $W^{u}{ }_{l o c}(q)$, we can suppose (after changing the values of the sequences $\left(m_{j}\right)$ and $\left(d_{j}\right)$ ) that we have instead

$$
f^{m_{j}}(x) \rightarrow p^{s} \in W^{s}(p) \backslash\{p\} \quad \text { and } \quad f^{d_{j}}(x) \rightarrow q^{u} \in W^{u}(q) \backslash\{q\} .
$$

By setting $x_{j}=f^{m_{j}}(x)$ and $k_{j}=d_{j}-m_{j}$ we can now apply the Connecting Lemma to obtain an $\varepsilon / 4$-perturbation $g$ of $f$ with $W^{s}\left(p^{g}\right) \cap W^{u}\left(q^{g}\right) \neq \emptyset$.

Since

$$
\begin{aligned}
\operatorname{dim}\left(W^{s}(p)\right)+\operatorname{dim}\left(W^{u}(q)\right) & =\operatorname{ind}(p)+(n-\operatorname{ind}(q)) \\
& >\operatorname{ind}(q)+n-\operatorname{ind}(q)=n
\end{aligned}
$$

where $n$ is the dimension of $M$, it follows that after another $\varepsilon / 4$-perturbation we obtain $g^{\prime} \in U$ such that the intersection $W^{s}\left(p^{g^{\prime}}\right) \cap W^{u}\left(q^{g^{\prime}}\right)$ is transversal at some point, and therefore persistent. Now, making another $\varepsilon / 4$-perturbation if necessary, we can assume that $g^{\prime}$ belongs to $R$, and therefore that $q^{g^{\prime}}$ belongs to $\Lambda\left(p^{g^{\prime}}\right)$.

Finally, to obtain an intersection between $W^{u}(p)$ and $W^{s}(q)$ after an $\varepsilon / 4$-perturbation $f^{\prime}$ of $g^{\prime}$, we use the same argument as above. The end result is an $\varepsilon$-perturbation of $f$ that has a heterodimensional cycle between the continuations of $p$ and $q$, as desired.

Proof of Theorem C. - Set $R=R_{+} \cap R_{5}$ with $R_{+}$as in Corollary B.2 and $R_{5}$ as in Theorem 5. Consider $f \in R$ with finitely many homoclinic classes and finitely many periodic sinks/sources. Let $\Omega(f)=\Lambda\left(p_{1}\right) \cup \cdots \cup \Lambda\left(p_{k}\right)$ be the spectral decomposition of $f$ as in Proposition A. Assume that $f$ is not omega-stable. By Theorem 6 this implies that $f$ does not belong to $\Im^{1}(M)$. Choose some $\varepsilon>0$. To prove the theorem we must create a heterodimensional cycle after an $\varepsilon$-perturbation of $f$.

Choose pairwise disjoint open neighborhoods $W_{1}, \ldots, W_{k}$ of $\Lambda\left(p_{1}\right), \ldots, \Lambda\left(p_{k}\right)$ respectively. Then there exists a neighborhood $U$ of $f$ in $\operatorname{Diff}^{1}(M)$ that satisfies conditions (i) and (ii) of Corollary B.2. Since $U$ is an open neighborhood of $f$ and $f$ does not belong to $\Im^{1}(M)$, it follows that there exists some $g$ in $U, \varepsilon / 4$-close to $f$, with some nonhyperbolic periodic point $q$. Since $q$ is periodic, it is a nonwandering point of $g$, and therefore $q$ belongs to $W_{1} \cup \cdots \cup W_{k}$. 
Let $W_{j}$ be the neighborhood that contains $q$ and let $s$ be the stability index of $p_{j}$. Since $q$ is nonhyperbolic (i.e., at least one of its eigenvalues has norm 1), we can apply Franks' Lemma to the orbit of $q$, creating after an $\varepsilon / 4$-perturbation $g^{\prime} \in U$ of $g$ a hyperbolic periodic point $q^{\prime}$ of $g^{\prime}$ whose $g^{\prime}$-orbit coincides with the $g$-orbit of $q$ and whose stability index is $\neq s$. This "new" hyperbolic periodic point $q^{\prime}$ cannot be a source/sink by Theorem 5; hence $q^{\prime}$ is a saddle.

Now $q^{\prime}$ is a hyperbolic periodic point of $g^{\prime}$, so it persists and varies continuously near $g^{\prime}$. In particular, for $g^{*}$ close enough to $g^{\prime}$, the continuation $q^{*}$ of $q^{\prime}$ still belongs to $W_{j}$. So after an $\varepsilon / 4$-perturbation $g^{*}$ of $g^{\prime}$ we have $g^{*} \in(U \cap R)$ and $q^{*}$ still in $W_{j}$. Since by hypothesis $\left(W_{j} \cap \Omega\left(g^{*}\right)\right)=\Lambda\left(p_{j} g^{*}\right)$, it follows that $q^{*}$ belongs to $\Lambda\left(p_{j}{ }^{*}\right)$. But remember that

$$
\operatorname{ind}\left(q^{*}\right) \neq s=\operatorname{ind}\left(p_{j}{ }^{*}\right)
$$

so $\Lambda\left(p_{j}{ }^{g^{*}}\right)$ cannot be the orbit of a sink/source, hence $p_{j} g^{*}$ is necessarily a saddle and $\Lambda\left(p_{j} g^{*}\right)$ is its homoclinic class.

Thus $\Lambda\left(p_{j}{ }^{*}\right)$ contains periodic saddles of different indices at $g^{*}$. Moreover, this occurs in a locally residual neighborhood of $g^{*}$. To see this, taking if necessary a smaller neighborhood of $g^{*}$, we can assume that $q^{*}$ has a continuation in all of $U$. Again shrinking $U$ if necessary, we can assume that the continuation of $q^{*}$ belongs to $W_{j}$ for all diffeomorphisms in $U$. Then for all $h \in(U \cap R)$ we have that $\left(q^{*}\right)^{h} \in\left(W_{j} \cap \Omega(h)\right)=\Lambda\left(p_{j}{ }^{h}\right)$, so $\Lambda\left(p_{j}{ }^{h}\right)$ contains two periodic saddles of different indices (namely, $\left(q^{*}\right)^{h}$ and $\left.p_{j}{ }^{h}\right)$. Therefore $\Lambda\left(p_{j}{ }^{g^{*}}\right)$ satisfies the hypotheses of Lemma 2 above.

We now apply Lemma 2 to $\Lambda\left(p_{j}{ }^{*}\right)$ to create, after yet another $\varepsilon / 4$-perturbation, a heterodimensional cycle between the continuations of $q^{*}$ and $p_{j}{ }^{g^{*}}$. Having gone through 4 successive $\varepsilon / 4$-perturbations of $f$, we have obtained a heterodimensional cycle via an $\varepsilon$-perturbation of $f$, as desired.

\section{Acknowledgements}

The support and encouragement of my thesis advisor Marcelo Viana was crucial during the writing of this paper; so was the stimulating research environment at IMPA. Thanks also to Christian Bonatti and to Lorenzo Diaz for valuable conversations and remarks.

\section{REFERENCES}

[1] ABDEnUR F., Attractors of generic diffeomorphisms are persistent, preprint IMPA, 2001.

[2] Bonatti Ch., Diaz L.J., Persistence of transitive diffeomorphisms, Ann. Math. 143 (1995) 367-396.

[3] Bonatti Ch., Diaz L.J., Connexions hétéroclines et généricité d'une infinité de puits ou de sources, Ann. Scient. Éc. Norm. Sup. Paris 32 (1999) 135-150.

[4] Bonatti CH., Diaz L.J., On maximal transitive sets of generic diffeomorphisms, preprint PUC-Rio, 2001.

[5] Bonatti Ch., Diaz L.J., Pujals E., A $C^{1}$-generic dichotomy for diffeomorphisms: weak forms of hyperbolicity or infinitely many sinks or sources, Ann. Math., to appear.

[6] Bonatti Ch., Diaz L.J., Pujals E., Rocha J., Robustly transitive sets and heterodimensional cycles, Astérisque, to appear.

[7] Bonatti CH., Viana M., SRB measures for partially hyperbolic systems whose central direction is mostly contracting, Israel J. Math. 115 (2000) 157-193.

[8] Carballo C.M., Morales C.A., Homoclinic classes and finitude of attractors for vector fields on n-manifolds, preprint, 2001.

[9] Carballo C.M., Morales C.A., PaCifico M.J., Homoclinic classes for generic $C^{1}$ vector fields, Ergodic Theory Dynam. Systems, to appear. 
[10] Diaz L.J., Pujals E., URes R., Partial hyperbolicity and robust transitivity, Acta Math. 183 (1999) $1-43$.

[11] FrANKS J., Necessary conditions for stability of diffeomorphisms, Trans. AMS 158 (1971) 301-308.

[12] Hayashi S., Diffeomorphisms in $\Im^{1}(M)$ satisfy Axiom A, Ergodic Theory Dynam. Systems 12 (1992) 233-253.

[13] HaYAshi S., Connecting invariant manifolds and the solution of the $C^{1}$ stability and $\Omega$-stability conjectures for flows, Ann. Math. 145 (1997) 81-137.

[14] Kelley J.L., General Topology, New York, Springer, 1955.

[15] MAÑ́ R., Contributions to the $C^{1}$-stability conjecture, Topology 17 (1978) 386-396.

[16] Mañé R., An ergodic closing lemma, Ann. Math. 116 (1982) 503-540.

[17] PALis J., A global view of dynamics and a conjecture on the denseness of finitude of atttractors, Astérisque 261 (2000) 335-347.

[18] Pugh C., An improved closing lemma and a general density theorem, Amer. J. Math. 89 (1967) 10101021.

[19] Pujals E., Sambarino M., Homoclinic tangencies and hyperbolicity for surface diffeomorphisms: a conjecture of Palis, Ann. Math. 151 (2000) 961-1023.

[20] Palis J., Takens F., Hyperbolicity and Sensitive-Chaotic Dynamics at Homoclinic Bifurcations, Cambridge Univ. Press, 1993.

[21] Shub M., Global Stability of Dynamical Systems, Springer-Verlag, New York, 1986.

(Manuscrit reçu le 1er août 2001 ; accepté, après révision, le 3 juillet 2002.)

Flavio ABDENUR

IMPA,

Estrada D. Castorina 110,

Jardim Botânico, 22460-010 Rio de Janeiro RJ, Brazil

E-mail: flavio@impa.br 